Fluctuation and Noise Letters

Vol. 10, No. 4 (2011) 419-430

(C) World Scientific Publishing Company

DOI: $10.1142 / \mathrm{S} 0219477511000715$

\title{
ION TRAFFIC THROUGH A CELL MEMBRANE - AND HOW ITS $1 / f$ NOISE CONNECTS TO GAMBLER'S RUIN, CATALAN NUMBERS AND ZIPF'S LAW
}

\author{
MARTIN BIER \\ Department of Physics, East Carolina University \\ Greenville, North Carolina 27858, USA \\ bierm@ecu.edu \\ JILL GALLAHER \\ Integrated Mathematical Oncology, Moffitt Cancer Center \\ Tampa, Florida 33612, USA \\ jill.gallaher@moffitt.org \\ Received 30 March 2011 \\ Accepted 5 August 2011 \\ Communicated by S. M. Bezrukov
}

\begin{abstract}
$1 / f$ Noise has been observed in currents through ion channels, in currents through pores in lipid bilayers, and in the voltage noise of live cells. In the case of the ion channels and bilayer pores, a mechanism has been proposed and corroborated. The mechanism appears robust and may share an underlying logic with Zipf's Law and Gambler's Ruin.
\end{abstract}

Keywords: Electropore; lipid freezing; power law; nonequilibrium processes.

\section{Power Laws in the Kinetics of Ion Channels}

In the course of the 1970s, the idea became firmly established that it is ion channels that are behind a cell membrane's permeability to ions [1]. Ion channels are large membrane proteins and they are generally specific to just one kind of ion. The first models to describe the kinetics of an ion channel were simple $\mathrm{C} \rightleftharpoons \mathrm{O}$, i.e., the channel has a closed state $(\mathrm{C})$ and an open state $(\mathrm{O})$. The two rates between the states are constant in time. These rates can depend on parameters like $\mathrm{pH}$, ligand concentration or membrane potential. It is because of this that the passive flow of ions can be regulated.

The Markovian $\mathrm{C} \rightleftharpoons \mathrm{O}$ scheme with its constant rates predicts exponentially distributed dwelling times in both states, i.e., $f(t) \propto \exp [-k t]$, where $f(t)$ is the probability density for the duration of the open or closed state and $k$ is the rate out of that state. A Markovian $\mathrm{C} \rightleftharpoons \mathrm{O}$ scheme, furthermore, leads to a power spectrum for the fluctuating current that is Lorentzian $[1,2]$. The power spectrum $S(\omega)$ is 
useful as it describes how the energy in the $\mathrm{C} \rightleftharpoons \mathrm{O}$ fluctuation is distributed over the different frequencies. $S(\omega) d \omega$ is proportional to the power that the signal carries between the frequencies $\omega$ and $\omega+d \omega$. For $\mathrm{C} \rightleftharpoons \mathrm{O}$, we have $S(\omega) \propto\left(\omega_{c}^{2}+\omega^{2}\right)^{-1}$, where $\omega_{c}$ is the characteristic rate or relaxation rate of the $\mathrm{C} \rightleftharpoons \mathrm{O}$ scheme, i.e., $\omega_{c}=k_{C O}+k_{O C}[1,2]$. With a log scale for the frequency, this simple Lorentzian power spectrum looks like two plateaus, where the "jump" from the higher plateau to the lower plateau at $S(\omega)=0$ occurs at the characteristic rate of the system.

Single channel patch clamp experiments showed that, in many cases, the open and closed time distributions looked more like a power law, i.e., $f(t) \propto t^{-\alpha}$, where $\alpha$ is a constant [3]. Power spectra of such ion channels commonly exhibited no characteristic rate, but, instead, $1 / f$ behavior (i.e., $S(\omega) \propto 1 / \omega^{\gamma}$, where $\gamma$ is close to unity) over a significant frequency range. For many channel data, it is possible to come up with an ad hoc fix and fit experimental results to reasonable satisfaction with schemes like $\mathrm{C} \rightleftharpoons \mathrm{O} \rightleftharpoons \mathrm{O}$ or $\mathrm{C} \rightleftharpoons \mathrm{O} \rightleftharpoons \mathrm{C}$ or even more complicated ones. But on a more fundamental level it has been suggested that reality may be better described by a $\mathrm{C} \rightleftharpoons \mathrm{O}$ scheme, where the transition rates decrease in time like $k(t) \propto t^{-\beta}$. The underlying idea is the following. A protein is a very complicated molecule and, instead of it leaping between a few conformational states, it should be imagined as diffusing in many dimensional conformational spaces [3]. Going from a closed state $\mathrm{C}$ to an open state $\mathrm{O}$, the channel crosses an activation barrier in its conformational space. Along a one-dimensional reaction coordinate, the energy profile can be depicted as follows:

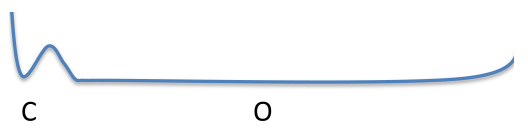

The position of a Brownian particle in this potential would represent the state of the protein. So, right after opening, when the imagined particle is still very close to the barrier, the probability to close again is very high. Over time, the average position of the particle moves further and further away from the barrier and the rate for the $\mathrm{O} \rightarrow \mathrm{C}$ transition consequently decreases. The aforementioned $k_{O C}(t) \propto t^{-\beta}$ thus results. To describe the diffusive behavior of a particle in this potential, one takes a Markov system

$$
\mathbf{C} \leftarrow \mathbf{O}_{1} \rightleftharpoons \mathbf{O}_{2} \rightleftharpoons \mathbf{O}_{3} \rightleftharpoons \cdots \rightleftharpoons \mathbf{O}_{N}
$$

where the larger subscript indicates a larger distance from the barrier. Here, all the rates are taken to be identical and equal to $\lambda$. In measuring a current through an ion channel, the digital sampling takes place at a finite rate. It is, furthermore, common to apply a filter for the suppression of noise. Because of the sampling and filtering, it is legitimate to describe the diffusive behavior in potential (1) by the kinetic scheme depicted in (2), and take the filter frequency as the rate $\lambda$. This is because events with a timescale faster than $1 / \lambda$ will not be observed anyway. 
After absorbing $\lambda$ into the timescale (i.e., taking all the rates equal to 1 ), and writing $p_{n}(t)$ for the probability that the system is in state $\mathrm{O}_{n}$ at time $t$, we can set up the following master equations associated with scheme (2):

$$
\begin{aligned}
& \dot{p}_{1}(t)=-2 p_{1}+p_{2} \\
& \dot{p}_{n}(t)=p_{n-1}-2 p_{n}+p_{n+1} \quad \text { for } 1<n<N \\
& \dot{p}_{N}(t)=p_{N-1}-p_{N} .
\end{aligned}
$$

We imagine that an opening, i.e., a transition from $\mathrm{C}$ to $\mathrm{O}_{1}$, occurs at $t=0$. So we have $p_{1}(0)=1$ and $p_{n}(0)=0$ for $n \geq 2$. The probability $P(t)$ for the channel to remain open at time $t$ equals:

$$
P(t)=\sum_{n=1}^{N} p_{n}(t) .
$$

In the analysis of experimentally obtained open-closed recordings, it is common to depict the distribution of open times in a histogram. When normalized, such a histogram is a probability density function $f(t)$ of open times. The aforementioned $P(t)$ represents the probability that the pore stays open till a time larger than $t$ (i.e., $\left.P(t)=\int_{t}^{\infty} f(\tau) d \tau\right) . P(t)$ and $f(t)$ are thus related through $f(t)=-\dot{P}(t)=p_{1}(t)$. It is actually possible to obtain an analytic solution of (3) in terms of modified Bessel functions. However, our concern is with the large $t$ asymptotic behavior. After undoing the absorption of $\lambda$ into the timescale, it is found that:

$$
f(t) \approx \frac{t^{-3 / 2}}{2 \sqrt{\pi \lambda}}
$$

This approximation applies when $t \gtrsim(1 / \lambda)$. Since the experiments do not cover the shorter timescales anyway, this is the formula against which the obtained histograms are to be checked. This result is also shown in the references [4] and [5].

The distribution $f(t)$ is related to the transition rate $k(t)$ out of the open state in the following way: $k(t)=f(t) / P(t)=-\frac{d}{d t} \ln P(t)$. This leads to $k(t) \approx(2 t)^{-1}$. The relation $k(t) \approx(2 t)^{-1}$ is the same in scaled and in unscaled units of time.

The robustness of the $t^{-3 / 2}$ asymptotic behavior when the system is tweaked has been researched extensively [6]. In a series of articles in the early 2000s, Goychuk and Hänggi returned to the potential depicted in (1) [7-9]. They analyzed the diffusive motion with a Fokker-Planck equation. They again found the same asymptotic behavior described in Eq. (5). Even if the O-state in (1) is not perfectly flat but instead, exhibits some minor variations, the same power laws ensue. All in all, the derived "-3/2" asymptotic behavior of Eq. (5) appears to be very stable under small perturbations.

In the final sections of this article, we will extensively discuss the $-3 / 2$ power law. We will see how the above derived $-3 / 2$ originates in a structure that is more universal than just the asymptotics of the potential shown in (1). 


\section{2. $1 / f$ Noise from Lipid Bilayer Pores at the Freezing Transition}

Around the same time that the existence of ion channels became firmly established, some curious properties of the lipid bilayer membrane were discovered.

The lipid bilayer, in which the ion channels are embedded, constitutes the main part of the cell membrane. The image on the far left of Fig. 1 shows the structure of the lipid bilayer under "normal" conditions. The polar phospholipid headgroups are sticking out into the aqueous solutions on the intra- and extracellular side. Apolar tails consisting of chains of about 15 carbons are pointed towards each other. The cell membrane is only about 5 nanometers thick, and it is held together by hydrophobic and hydrophilic forces. Water passes through the cell membrane easily, but "normally" the bilayer structure is almost completely impermeable to ions.

In 1973, experimental work by Papahadjopoulos et al. showed that the permeability to ions of the lipid bilayer membrane exhibits a sharp peak right at the melting transition [10]. Under physiological conditions, the cell membrane is a $2 \mathrm{D}$ liquid, i.e., phospholipids diffuse freely in the plane of the membrane. When frozen, the lipid tails get entangled with and bound to each other. We then effectively get a gel in which the individual phospholipids no longer diffuse. In the same way that near $0^{\circ} \mathrm{C}$ blocks of ice can float in water, we can have solid rafts "floating" in the liquid membrane near the phase transition temperature. These rafts can rapidly shrink and grow. Because the freezing point of a cell membrane is generally only a few degrees below the physiological temperature, the study of the enhanced ion permeability of a cell membrane near the phase transition carries biological significance.

On the molecular level, Brownian fluctuations in the free energy $E$ are of the order of $k_{B} T$, where $k_{B}$ represents Boltzmann's constant and $T$ is the absolute temperature. The solid-to-liquid phase transition involves a large change in entropy $S$. A large change in entropy must be accompanied by a large change in the enthalpy $H$ as $E=H-T S$. Through Kubo's fluctuation theorem, i.e., $c_{P}=\left(\left\langle H^{2}\right\rangle-\langle H\rangle^{2}\right) / k_{B} T^{2}$, it can be understood how these increased enthalpy fluctuations are related to the increased $c_{P}$ (the heat capacity at constant pressure) at the phase transition $[11,12]$. Also, the volume and the surface area change by a few percent when melting occurs.

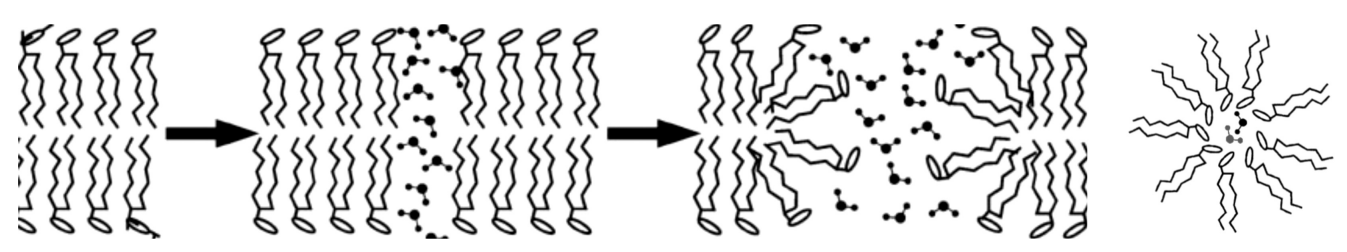

Fig. 1. The likely scenario for the formation of a pore in a lipid bilayer membrane. Through a random fluctuation, an open corridor first develops. Next, phosphate headgroups reorient to form a polar lining that facilitates easy flow of water and ions through the pore. The drawing on the far right is a top view of the pore. The other drawings are cross sections through the center of the pore. The figure is adapted from [36]. 
In computer simulations of a lipid bilayer membrane at the phase transition, it has been shown that fluctuations in the liquid are particularly large in the vicinity of the solid-liquid interface [12]. It is thus near these interfaces that pore formation will most likely occur. Figure 1 shows how, for a pore to form, it is necessary for phospholipids to first "fluctuate" away from each other. Subsequently, phospholipids can reorient and the phosphate headgroups can collapse into the lining of the pore. The hydrophilic pore lining next allows for the easy passage of water and ions. The two images on the right of Fig. 1 show a side view and a top view of the pore, respectively.

In order for a pore in a lipid bilayer to allow for the passage of water and small ions, it has to exceed a diameter of about $0.5 \mathrm{~nm}$. On the other hand, the creation of a pore structure as on the right of Fig. 1 requires an investment of energy. The cross sectional side view shows how, at the lining of a pore, the surface is highly curved and how the ends of the tails are crowded together. Making a larger diameter would create more pore edge and would require more energy [13]. The hydrophilic ionconducting pore is therefore most likely going to be at the minimal possible radius that allows for such conduction. Such a fixed conductance level is indeed what is observed. Records of electropores in lipid bilayers near the freezing transition look very much like ion channel records - there appear to be fluctuations between an open state and a closed state $[5,12,14-16]$.

In the liquid state, a pore generally rapidly closes again [13]. But near the phase transition and especially near a solid raft, the phospholipids in the pore lining may freeze. Such freezing would stabilize the pore. The freezing of more and more phospholipids around the pore would correspond to moving to the right in the open state in (1) and (2). For a pore to be able to seal again, the phospholipids should all melt again, i.e., the system should move back to $\mathrm{O}_{1}$.

Figure 2 shows n open time histogram for a pore in a lipid bilayer membrane. The reader is referred to [5] and [17] for the details on how the data and the figure were obtained. The straight line in Fig. 2(a) represents the prediction in Eq. (5). Not only do we see the predicted $-3 / 2$ slope in the graph, even the prefactor of Eq. (5) is borne out. Figure 2(b) shows the associated power spectrum of the electropore. In [18], it is shown how fluctuations between the open and the closed state follow $S(\omega) \propto 1 / \omega^{1 / 2}$ if both the closed and the open state have an underlying Markovian array (and the associated $f(t) \approx\left(2 \sqrt{\pi \lambda} t^{3 / 2}\right)^{-1}$ ) like our open state in (1) and (2). A straight line with the predicted slope of $-1 / 2$ is drawn in Fig. 2(b) for reference. The data in Fig. 2 were taken with a $300 \mathrm{~Hz}$ filter. So no phenomenon faster than about $3 \mathrm{~ms}$ can be observed. It is because of this that the data points appear to "collapse" at a timescale faster than $3 \mathrm{~ms}$. As data were taken for less than a minute, there is also an obvious cutoff for the lower frequencies.

"Dangling ends" of the polymers in the lipid bilayer moving into the center of the pore and blocking the flow of water and ions have been offered as an explanation for the observed $1 / f$ noise in biological [19] and artificial channels [20]. Such blocking by dangling ends can well follow the kinetics we described above with (1) and (2). 


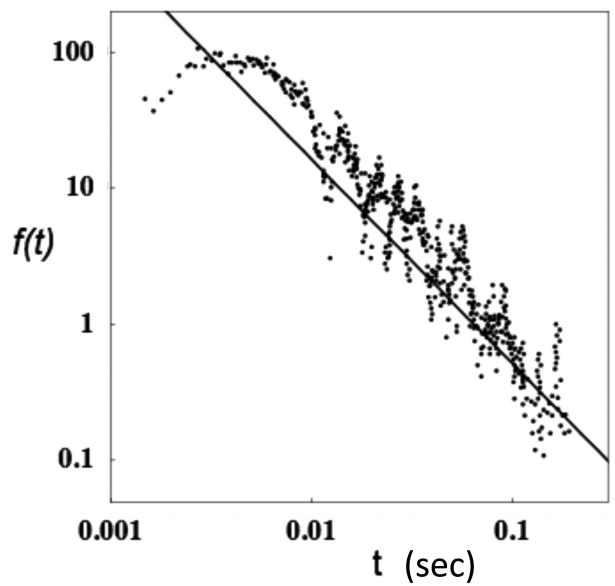

(a)

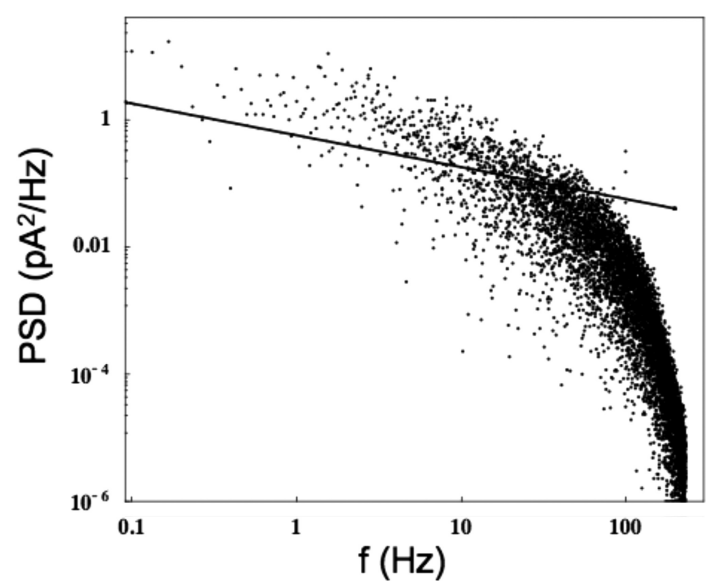

(b)

Fig. 2. From [5], an open time histogram (2a) and a power spectrum (2b) for an electropore in a lipid bilayer. The straight line in (2a) is the theoretical prediction Eq. (5). The straight line in (2b) has predicted slope $-1 / 2$ and is for reference. Details on how data were obtained and processed can be found in Refs. [5] and [17].

If, after initial blockage, the further moving of the polymer into the center of the pore does not involve any significant change in energy, then such "further moving" would be like a diffusing away from the activation barrier as in (1). This would lead to a " $-3 / 2$ " power law distribution (cf. Eq. (5)) for also the closed times. And it would explain the observed $-1 / 2$ slope in Fig. 2(b). Also the diffusion of more dangling ends into the pore's center after the initial blockage by just one dangling end, could be described with kinetics similar to (1) and (2). After all, if two polymer ends are blocking the flow through a pore, they both have to pull back into the lining for the pore to be open again.

\section{3. $1 / f$ Noise Across the Membrane of a Living Cell}

In the mid-1960s, Verveen and Derksen recorded 5-10 minutes of cell membrane electrical noise at a Ranvier node of an unstimulated nerve cell [21]. For myelinated nerve cells the node of Ranvier is where the action potential is generated. There is a high concentration of ion channels there and, in the days before patch clamp, it was a good place to measure electrical activity. What they found was $1 / f$ noise over about two decades (between $10 \mathrm{~Hz}$ and $1000 \mathrm{~Hz}$ ). $S(\omega) \propto \omega^{-1}$ was a remarkably good fit.

In 2004, Diba et al. utilized much improved equipment to replicate this result [22]. In the article by Diba et al., it is pointed out that there are many different ion channels present in the cell membrane with many different characteristic rates. They argue that the $1 / f$ appearance comes about as the cumulative effect, i.e., the sum of many individual Lorentzians. The argument echoes that of Bernamont [23]. 
In 1937, Bernamont explained $1 / f$ noise in electrical currents as a consequence of a variable rate for the electron's transition rate from the conduction band to a bound state. He assumed that the rate would depend on the speed of the flowing electron.

It seems unlikely that, in so many experiments and setups, amplitudes and characteristic times of different Lorentzians would, time and time again, "conspire" to yield the $1 / f$ appearance after these Lorentzians are put together. Mechanisms like the one in the above system (2) may be behind many instances of $1 / f$ noise. The aforementioned $1 / f$ noise found with electropores in the lipid bilayer, may well contribute significantly to the $1 / f$ noise across the membrane of a living cell.

\section{Combinatorics Behind the "-3/2" Power Law}

To see the universality of the $-3 / 2$ power law, we need to go back to scheme (2). The master equation (3) that ensues from (2) is set up with the Markov assumption, i.e., the idea that transition rates are constant. We thus end up dealing with flows that are described by a set of coupled linear differential equations. Such continuous dynamics is not much different from a discrete dynamics where we let time run in fixed steps and at each step, an imagined particle has to go to either the left or the right. That the asymptotics for both systems have to be the same can be understood as follows.

The $f(t)$ in Eq. (5) describes how, for large open times $t$, the frequency of occurrence of such open times decreases with $t$. For large $t$ 's many transitions between different states are made in the system depicted in scheme (2). The individual transitions are Markovian and the dwell time in each of the states has an exponential distribution. Consider a transition $\mathrm{X}_{1} \rightarrow \mathrm{X}_{2}$ with a rate $k$. If a particle is in state $\mathrm{X}_{1}$ at $t=0$, then the average time after which transition occurs equals $1 / k$. It is easy to prove that for an exponential distribution of transit times, this average equals the standard deviation. So the standard deviation also equals $1 / k$. The variance is the square of the standard deviation and equals $(1 / k)^{2}$. Going back to scheme $(2)$, we realize that for the longer open times, many transitions between the different states are involved. If all the rates are equal to 1 (which leads to $f(t) \approx\left(2 \sqrt{\pi} t^{3 / 2}\right)^{-1}$ ), then the average time in any state equals $1 / 2$. This is because every open state in scheme (2) has two outgoing transitions, so effectively $k=2$ for the outgoing rate. For an opening involving $m$ steps within the sequence of O-states in scheme (2), the average open time will be $m\left(\frac{1}{2}\right)$. For subsequent stochastic processes, the variances add up. So the variance in the open times will be $m\left(\frac{1}{2}\right)^{2}$ and the standard deviation will be $\sqrt{m}\left(\frac{1}{2}\right)$. It is obvious that for long open times (large $m$ and large $t$ ), the standard deviation becomes negligible in comparison to the average. So, the limit of the Markovian system behaves clockwork-like and can be described with the proposed discrete system.

For the proposed discrete stepper, it is possible to simply list the sequences of steps and identify the number of steps with the open time of the pore. But before 
Table 1. The Catalan numbers, $C_{n}$ and how they relate to system (2). "R" corresponds to a step to the right in the kinetic scheme (2) and " $L$ " corresponds to a step to the left. The first " $R$ " in each sequence is the initial step from state $\mathrm{C}$ to state $\mathrm{O}_{1}$. " $m$ " denotes the total combined number of R's and L's in a sequence. " $n$ " equals $m / 2-1$ and enumerates the Catalan numbers $C_{n} . C_{n}$ gives the number of possible sequences of $m$ symbols. With a residence time of $1 / 2$ in each state, $t=n+\frac{1}{2}=\frac{1}{2}(m-1)$ gives the total duration of an opening that corresponds to a sequence of $m$ steps.

\begin{tabular}{ccclc}
\hline$n$ & $m$ & $t$ & Possible permutations & $C_{n}$, \# of permutations \\
\hline 0 & 2 & $\frac{1}{2}$ & RL & 1 \\
1 & 4 & $\frac{3}{2}$ & RRLL & 1 \\
2 & 6 & $\frac{5}{2}$ & RRRLLL, RRLRLL & 2 \\
3 & 8 & $\frac{7}{2}$ & RRRRLLLL, RRRLRLLL, & 5 \\
& & & RRRLLRLL, RRLRRLLL, & \\
& & & RRLRLRLL & \\
. &. &. &. &. \\
. &. &. &. &. \\
. &. &. &. & \\
\hline
\end{tabular}

starting that endeavor, it is important to realize again that there are two transitions out of any open state in the system depicted in (2). If both these transitions have a rate that equals unity, then the average time after which a step occurs equals $1 / 2$. In Table 1, $m$ denotes the number of transitions involved in an opening. It is obvious that only even $m$ 's are allowed. The variable $n=m / 2-1$ ranks the open times. $\mathrm{R}$ denotes a step to the right and $\mathrm{L}$ denotes a step to the left. The first $\mathrm{R}$ corresponds to the $0 \rightarrow 1$ transition that commences a pore opening. After that transition, a time of $1 / 2$ is spent in each state. So we get $t=n+1 / 2=\frac{1}{2}(m-1)$ for the total open time.

For a particular sequence of $m$ symbols, the probability of that sequence to occur equals $\left(\frac{1}{2}\right)^{m-1}=\left(\frac{1}{2}\right) 4^{-n}=\left(\frac{1}{2}\right) 4^{-t+(1 / 2)}=4^{-t}$. There is a " -1 " in the $(m-1)$ exponent because an opening always starts with an R. Subsequently, each transition is like the result of a coin toss where each outcome has a probability of $1 / 2$. A particular sequence of duration $t$ thus has a probability that decreases exponentially with $t=n+1 / 2$. We will next see that a power law eventually emerges because this exponential decrease is compensated for by an exponential increase with $n$ of the number of permutations.

With Table 1, the central question is: how does the number of permutations exactly increase with $n$ ? Fortunately, many problems in applied science have boiled down to the identical combinatorics problem. [24] is a textbook that lists 80 such problems in applied science. A classic example is the following. If we associate $\mathrm{R}$ with a left parenthesis, i.e., "(", and L with a right parenthesis, i.e., ")", then the sequences in Table 1 correspond to legitimate nestings of matching parentheses. A mathematical formalization of this is called a Dyck Language [25]. The numbers of allowed permutations are named the "Catalan Numbers" $\left(C_{n}\right)$, after the 19th 
century Belgian mathematician Eugène Catalan:

$$
C_{n}=\frac{1}{n+1}\left(\begin{array}{c}
2 n \\
n
\end{array}\right) \text {. }
$$

The Wikipedia page on Catalan Numbers [26] offers five ingenious proofs of this formula. We are interested in the $n \rightarrow \infty$ limit and, for that purpose, we apply Stirling's approximation $\left(n ! \approx \sqrt{2 \pi n}\left(\frac{n}{e}\right)^{n}\right)$. We then find

$$
C_{n} \approx \frac{4^{n}}{n^{3 / 2} \sqrt{\pi}}
$$

The probability for an $n$-rank opening (which implies that there are $m=2 n+2$ steps involved) equals $p_{n}=\frac{1}{2} C_{n} 4^{-n}$. With $t=n+\frac{1}{2}$, this leads to $p_{t}=C_{n} 4^{-t}$. Substituting Eq. (7) and $n=t-1 / 2$, we find in the $t \rightarrow \infty$ limit

$$
p_{t} \approx \frac{1}{2 \sqrt{\pi}} t^{-3 / 2}
$$

which is exactly the asymptotic behavior that we derived earlier for the associated Markov system.

\section{A Connection to Zipf's Law}

One could think of the sequences in the fourth column of the Table 1 as words in a 2-letter alphabet. Elaborating on this idea, we can get some insights in Zipf's Law. In 1932, George Kingsley Zipf first formulated this empirical law [27] and it has puzzled researchers ever since. Given a large amount of text in any language, it is possible to rank words from the most frequently occurring to the least frequently occurring. If one then graphs the logarithm of that frequency versus the logarithm of the rank, the data are found to follow a straight line with a slope close to -1 , i.e., $f(r) \propto r^{-1}$, where $f$ is the frequency and $r$ is the rank. Zipf's Law appears to hold in many different languages. It also appears to hold when craters on the moon are ranked according to their size or when cities in a country are ranked according to their population sizes. Zipf's Law can be distilled from the data in many very different systems: DNA sequences, internet traffic, etc. [28] is a website where hundreds of articles on the subject are brought together. But Zipf's Law is still very much an empirical discovery. Many attempts have been made to formulate basic underlying dynamics that give rise to Zipf's Law. Ad hoc explanations have been found to apply in some cases [29]. No satisfying general theory, however, has been formulated.

In his characteristically cryptic manner, Mandelbrot shows, in Chapter 38 of his groundbreaking "The Fractal Geometry of Nature," that a kind of Zipf's Law emerges when a random generator is allowed to produce strings using $M$ letters and one space [30]. In such a setup, a word of $N$ letters will always occur more frequently than a word of $N+1$ letters. So word rank can then be equated with word length. In 1992, Wentian Li worked out the details of the associated statistics [31]. He let the 
space and the symbols of the $M$-letter alphabet have equal probability and deduced Zipf's power law: $f(r) \propto r^{-\alpha}$, where $\alpha=\ln (M+1) / \ln M$. It is obvious that for the customary 26 -letter alphabet, $\alpha$ will be very close to one. Li's result could be taken to indicate that Zipf's Law is purely a consequence of random statistics. However, one can also take it as an affirmation of Zipf's "Principle of Least Effort" [32], i.e., support for the idea that language evolution has maximized efficiency in the sense that the words that we need the most often are also the shortest words.

For $M=2$, i.e., a two letter alphabet with a space, Li's result implies $\alpha=1.58$. There are minor, "dialectal," differences between our two-letter language (Table 1) and the Li's two-letter language. Not unlike natural languages, our case has restrictions. In our case, there are always more R's than L's as the word "grows." Our words end when, with the last letter, the number of L's has caught up with the number of R's. That a slight difference in the rules for word formation leads to slightly different powers, 1.50 versus 1.58 , is not surprising.

\section{Gambler's Ruin, $1 / f$ Noise, and Zipf's Law}

The kinetic scheme (2) also models a form of "Gambler's Ruin." In the 17th century, Christiaan Huygens initiated the study of games of chance and he researched problems like the following:

Let a player be given an amount of money $M$ by the house. A fair coin toss (50\% chance of winning and $50 \%$ chance of losing) determines whether he gets an extra $M$ or whether he loses $M$. The game is repeated as long as our player has money to play with. At every trial, the same amount $M$ is betted. It is obvious that the initial state is $\mathrm{O}_{1}$. Next, every win is a transition to the right (R) and every loss is a transition to the left $(\mathrm{L})$. If the house has an infinite amount of money, then the player is guaranteed to eventually lose his money. The duration of the game (or the "opening") may be infinitely long and the average duration, $\langle t\rangle=\int \tau f(\tau) \mathrm{d} \tau$, actually diverges for $f(\tau) \propto \tau^{-3 / 2}$. But it can be proven that $P(t)$ (cf. Eq. (4)) goes to zero as $t \rightarrow \infty$ and this means that a return to $\mathrm{C}$ (cf. (2)), i.e., the eventual loss of the initially donated amount of money $M$, is inevitable.

Standard references are not uniform in their definition of what constitutes $1 / f$ noise. For a power spectrum that behaves like $S(\omega) \propto 1 / \omega^{\alpha}, \alpha=0$ corresponds to white noise and $\alpha=2$ corresponds to brown noise. Brown noise is obtained when white noise is integrated over time to yield a simple random walk. The Wikipedia page [33] takes an in toto approach and labels the entire interval $0<\alpha<2$ as corresponding to $1 / f$ noise. The much cited paper by Milotti [34] is more restrictive in its recommendation: $1 / 2<\alpha<3 / 2$. By most definitions, the spectrum shown in Fig. 2(b), with $\alpha=1 / 2$, would qualify as $1 / f$ noise.

The electropore noise that we observe falls in a general category. Nonequilibrium systems, i.e., systems in which energy is dissipated, commonly exhibit $1 / f$ noise. Our electropore system is nonequilibrium as there is an electric potential between the two sides of the membrane that drives current through the pore whenever it is 
open. In spite of the ubiquitousness of $1 / f$ noise, no general theory has been formulated that convincingly explains all these $1 / f$ noise occurrences as a consequence of a universal mechanism [35]. Ad hoc explanations for particular instances is all that

physics has come up with. In this sense, $1 / f$ noise is very different from the white noise that we generally find in equilibrium systems and that can be understood as a consequence of Brownian motion. The Johnson-Nyquist noise that resistors exhibit when there is no imposed current or voltage, is a good example of equilibrium noise. Johnson-Nyquist noise is just a manifestation of the Brownian motion of charge carriers and of Einstein's fluctuation-dissipation relation. This is the reason that $k_{B} T$, the characteristic energy of Brownian motion, features prominently in the Johnson-Nyquist formula [35].

Zipf's Law is, in many ways, like $1 / f$ noise. Nonequilibrium dynamical processes (like evolution, language development, population dynamics, etc.) appear to commonly produce distributions that follow Zipf's Law. Like $1 / f$ noise, Zipf's Law has defied all attempts at the formulation of a general underlying theory. Its omnipresence is still somewhat of an enigma. With the kinetic scheme (2) and the ensuing "words" in Table 1, we have made explicit, for a particular case, that there is a link between $1 / f$ noise and Zipf's Law. That link may be indicative of a more general connection - a general connection that could be a fruitful venue of future research.

\section{Acknowledgment}

We are grateful to Larry Liebovitch for useful suggestions and discussions.

\section{References}

[1] B. Hille, Ionic Channels of Excitable Membranes (Sinauer, Sunderland, MA, 1992).

[2] L. J. DeFelice, Introduction to Membrane Noise (Plenum Press, New York, 1981).

[3] J. B. Bassingthwaighte, L. S. Liebovitch and B. J. West, Fractal Physiology (Oxford University Press, New York, 1994).

[4] G. L. Millhauser, E. E. Salpeter and R. E. Oswald, Proc. Natl. Acad. Sci. U.S.A. 85 (1988) 1503.

[5] J. Gallaher et al., Phys. Rev. E 81 (2010) 061925.

[6] C. A. Condat and J. Jäckle, Biophys. J. 55 (1989) 915.

[7] I. Goychuk and P. Hänggi, Proc. Natl. Acad. Sci. U.S.A. 99 (2002) 3552.

[8] I. Goychuk and P. Hänggi, Physica A 325 (2003) 9.

[9] I. Goychuk and P. Hänggi, Phys. Rev. E 70 (2004) 051915.

[10] D. Papahadjopoulos, K. Jacobson, S. Nir and T. Isac, Biophys. Acta 311 (1973) 330.

[11] R. Kubo, Rep. Prog. Phys. 29 (1966) 255.

[12] T. Heimburg, Thermal Biophysics of Membranes (Wiley-VCH Verlag, Weinheim, 2007).

[13] J. C. Weaver and Yu. Chizmadzhev, in Biological and Medical Aspects of Electromagnetic Fields, eds. F. S. Barnes and B. Greenebaum (CRC Press, Boca Raton, FL, 2006), p. 293.

[14] V. F. Antonov, V. V. Petrov, A. A. Molnar, D. A. Predvoditelev and A. S. Ivanov, Nature 283 (1980) 585. 
[15] A. Blicher, K. Wodzińska, M. Fidorra, M. Winterhalter and T. Heimburg, Biophys. J. 96 (2009) 4581.

[16] K. Wodzińska, A. Blicher and T. Heimburg, Soft Matter 5 (2009) 3319.

[17] J. A. Gallaher, Ph.D. Thesis, East Carolina University, Greenville, NC, 2010.

[18] S. B. Lowen and M. C. Teich, Phys. Rev. E 47 (1993).

[19] S. M. Bezrukov and M. Winterhalter, Phys. Rev. Lett. 85 (2000) 202.

[20] Z. Siwy and A. Fuliński, Phys. Rev. Lett. 89 (2002) 158101.

[21] H. E. Derksen and A. A. Verveen, Science 151 (1966) 1388.

[22] K. Diba, H. A. Lester and C. Koch, J. Neurosc. 24 (2004) 9723.

[23] J. Bernamont, Proc. Phys. Soc. 49 (1937) 138.

[24] R. P. Stanley, Enumerative Combinatorics, Vol. 2 (Cambridge University Press, 1999).

[25] M. Lothaire, Applied Combinatorics on Words (Cambridge University Press, 2005).

[26] http://en.wikipedia.org/wiki/Catalan_number (accessed March 29, 2011).

[27] G. K. Zipf, Selected Studies and the Principle of Relative Frequency in Language (Harvard University Press, Cambridge, MA, 1932).

[28] http://www.nslij-genetics.org/wli/zipf/ (accessed March 29, 2011).

[29] X. Gabaix, Quart. J. Econ. 114 (1999) 739-767.

[30] B. Mandelbrot, The Fractal Geometry of Nature (W. H. Freeman, San Francisco, 1983).

[31] W. Li, IEEE Trans. Inf. Theory 38(6) (1992) 1842.

[32] G. K. Zipf, Human Behavior and the Principle of Least-Effort (Addison-Wesley, Cambridge, MA, 1949).

[33] http://en.wikipedia.org/wiki/1_over_f_noise (accessed March 29, 2011).

[34] E. Milotti, arXiv:physics/0204033v1 (2002).

[35] J. C. Weaver and M. Bier, in Bioengineering and Biophysical Aspects of Electromagnetic Fields, eds. F. S. Barnes and B. Greenebaum (CRC Press, Boca Raton, 2007), p. 169.

[36] R. A. Böckmann, B. L. de Groot, S. Kakorin, E. Neumann and H. Grubmüller, Biophys. J. 95 (2008) 1837-1850. 